Schmerz 2015 $\cdot 29: 308$

DOI 10.1007/s00482-015-0010-8

Online publiziert: 29. Mai 2015

(c) Deutsche Schmerzgesellschaft e.V. Published by Springer-Verlag Berlin Heidelberg - all rights reserved 2015

F. Petzke' $\cdot$ P. Welsch ${ }^{2} \cdot$ P. Klose ${ }^{3} \cdot$ R. Schaefert ${ }^{4} \cdot$ C. Sommer ${ }^{5} \cdot$ W. Häuser ${ }^{6,7}$

${ }^{1}$ Schmerz-Tagesklinik und -Ambulanz, Universitätsmedizin Göttingen, Göttingen, Deutschland

${ }^{2}$ Stichting Rugzorg Nederland, Ede, Niederlande

${ }^{3}$ Abteilung für Naturheilkunde und Integrative Medizin, Kliniken Essen-Mitte, Essen, Deutschland

${ }^{4}$ Allgemeine und Innere Medizin, Universitätsklinikum Heidelberg, Heidelberg, Deutschland

${ }^{5}$ Neurologische Klinik, Universitätsklinikum Würzburg, Würzburg, Deutschland

${ }^{6}$ Klinik und Poliklinik für Psychosomatische Medizin und Psychotherapie,

Technische Universität München, München, Deutschland

${ }^{7}$ Innere Medizin I, Klinikum Saarbrücken gGmbH, Saarbrücken, Deutschland

\title{
Erratum zu: Opioide bei chronischem Kreuzschmerz
}

\section{Systematische Übersicht und Metaanalyse der Wirksamkeit, Verträglichkeit und Sicherheit in randomisierten, placebokontrollierten Studien über mindestens 4 Wochen}

Erratum zu:

Schmerz (2015) 29:60-72

DOI 10.1007/s00482-014-1449-8

In der Infobox 1 (Ausgeschlossene Studien) auf S. 70 fehlen zwei Literaturstellen.

Punkt 2 (Gordon et al.): Zu ergänzen ist Literaturstelle [34].

Punkt 4 (Yarlas et al.): Zu ergänzen ist: Alle Daten publiziert in [39].

Supplementary Material (English version):

In Infobox 1 (Excluded with reason), p. 9 , two reference numbers are missing. Item 2 (Gordon et al.): [34]

Item 4 (Yarlas et al.): All data published by [39]

Wir bitten um Beachtung.

\section{Korrespondenzadresse}

\section{PD Dr. W. Häuser}

Innere Medizin I

Klinikum Saarbrücken gGmbH

Winterberg 1, 66119 Saarbrücken

whaeuser@klinikum-saarbruecken.de 